Internist 2007 • 48:1432-1435

DOI 10.1007/s00108-007-1956-0

Online publiziert: 26. Oktober 2007

(c) Springer Medizin Verlag 2007

\section{Rubrikherausgeber}

K. Werdan, Halle/Saale
O. Tanneberger ${ }^{1} \cdot$ S. Büchner ${ }^{2} \cdot$ L.U. Zimmerli ${ }^{1}$

${ }^{1}$ Medizinische Poliklinik, Universitätsspital, Basel

${ }^{2}$ Dermatologische Klinik, Universitätsspital, Basel

\title{
Schnitzler-Syndrom mit Urtikaria-Vaskulitis
}

\section{Fallbericht}

\section{Anamnese}

Während eines Aufenthalts in Andalusien im Oktober 2001 stellte der 48-jährige ehemalige Flugkapitän ein urtikarielles Exanthem fest. Betroffen waren Oberschenkelinnenseiten, Hüfte, Rücken und Oberarme. Dieses Exanthem war initial begrenzt und im weiteren Verlauf konfluierend und löste juckende und brennende Missempfindungen aus. Zusätzlich traten Arthralgien und Abgeschlagenheit

\section{Liebe Leserinnen, liebe Leser. .}

in der Rubrik Kasuistiken präsentieren wir Ihnen interessante Fallberichte und ihre Lösungswege. Bei den vorgestellten Fällen handelt es sich entweder um ausgefallene Diagnosen oder um ungewöhnliche Verläufe häufiger Erkrankungen.

Lassen Sie Ihre Kolleginnen und Kollegen an Ihren Erfahrungen teilhaben und reichen Sie Ihre „Alltagsfälle", an die sich das evidenzbasierte diagnostische und therapeutische Vorgehen anschließen sollte, bei DER INTERNIST zur Publikation ein!

Wir freuen uns auf Ihre Fälle!

Bitte schicken Sie Ihre Kasuistiken direkt an den Rubrikherausgeber

Prof. Dr. K. Werdan

Klinikum Kröllwitz

Innere Medizin III

Ernst-Grube-Str. 40

06097 Halle/Saale

karl.werdan@medizin.uni-halle.de

Die Autorenrichtlinien finden Sie unter www.Derlnternist.de auf, einhergehend mit febrilen Temperaturen bis $39^{\circ} \mathrm{C}$. Diese komplexe Symptomatik hielt jeweils einige Stunden bis Tage an und wiederholte sich alle 3 Tage.

In der Anamnese war - neben einem Nikotinabusus - seit 4 Jahren eine ophthalmische Migräne ohne Kopfschmerzen bekannt. Familienanamnestisch wird ein Kolonkarzinom des Vaters berichtet.

\section{Klinischer Befund}

Bei der Untersuchung fielen mehrere 0,5$1,0 \mathrm{~cm}$ große, scharf begrenzte Makulae an den Oberschenkelinnenseiten auf, des Weiteren auch infiltrierte, urtikarielle, konfluierende, hellrote Herde dorsolumbal und an den Oberarmbeugeseiten. Zusätzlich zeigte sich eine $2 \times 3 \mathrm{~cm}$ große nummuläre Rötung über dem nicht geschwollenen rechten Malleolus lateralis.

Der internistische Status war unauffällig, insbesondere fanden sich keine Hepato- oder Splenomegalie und keine Lymphome.

\section{Diagnostik}

Labor. Hyperchromes, makrozytäres Blutbild mit Leukozytose (77\% Neutrophile und Lymphozytopenie von 16,5\%) und erhöhte Blutsenkungsgeschwindigkeit $(27 \mathrm{~mm} / \mathrm{h})$. In der Serumchemie leicht erhöhtes CRP (44 mg/l) sowie eine Hypercholesterinämie und erhöhte Leberwerte (ALAT ${ }_{43} \mathrm{U} / \mathrm{l}$ und $\gamma$-GT $176 \mathrm{U} /$ 1). TSH, Vitamin-B12- und Folsäurespiegel in der Norm. Rheumafaktor sowie ANA,

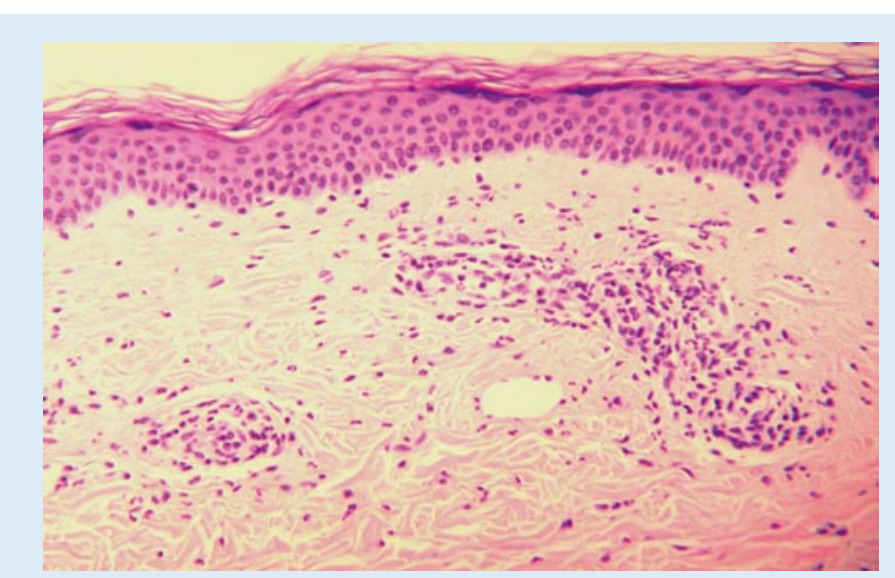

Abb. $1 \Delta$ Normale Epidermis mit unauffälliger dermoepithelialer Junktionszone. In der oberen Dermis zeigen sich perivaskuläre und interstitielle Infiltrate aus Lymphozyten, zahlreichen Neutrophilen und wenigen Eosinophilen. Die um die Gefäße angesammelten Granulozyten zerfallen teilweise unter Hinterlassung von Kerntrümmern (Leukozytoklasie). Interstitielles Ödem mit weit gestellten Lymphkapillaren 
Hier steht eine Anzeige.

第 Springer 


\section{Zusammenfassung · Abstract}

Internist 2007 · 48:1432-1435

DOI 10.1007/s00108-007-1956-0

(c) Springer Medizin Verlag 2007

O. Tanneberger · S. Büchner · L.U. Zimmerli

Schnitzler-Syndrom mit Urtikaria-Vaskulitis

Zusammenfassung

Das Schnitzler-Syndrom ist eine seltene Erkrankung, welche mit Urtikaria, periodischem Fieber, Knochenschmerzen bei Hyperostosis, Arthritiden und einer monoklonalen lgMGammopathie einhergeht. Es wurde 1974 durch die französische Dermatologin Liliane Schnitzler beschrieben. Aufgrund der sehr unterschiedlichen Symptome werden die Betroffenen oft von verschiedenen Fachärzten gesehen und sind insbesondere für Internisten, Rheumatologen, Hämatologen und Dermatologen von Interesse. Bislang war die Therapie oft schwierig und enttäuschend. Ein neuer therapeutischer Ansatz ist die Gabe eines Interleukin-1-Rezeptorantagonisten.

Schlüsselwörter

Schnitzler-Syndrom · Arthritis · Urtikaria · Periodische Fiebersyndrome - Vaskulitis

\section{Schnitzler's syndrome with urticaria vasculitis}

\begin{abstract}
Schnitzler's syndrome is a rare disease characterized by the association of chronic urticaria, intermittent fever, bone pain, arthritis, and monoclonal IgM gammopathy. It was first described by the French dermatologist Liliane Schnitzler in 1974. Because of the variety of clinical signs, the syndrome is of concern to doctors of different specialties and is of special interest to internists, rheumatologists, hematologists and dermatologists. Up to now, treatment was often difficult and disappointing. Interleukin-1 receptor antagonists offer a new therapeutic option.
\end{abstract}

Keywords

Schnitzler's syndrome · Arthritis · Urticaria . Periodic fever syndromes · Vasculitis

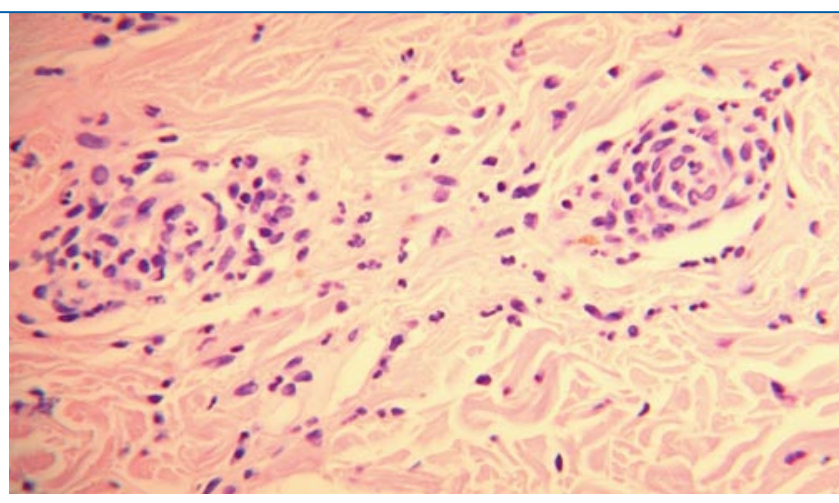

Abb. $2 \varangle$ Postkapilläre Venolen im Korium zeigen eine Schwellung und sind von neutrophilen Granulozyten durchsetzt

extrahierbare nukleäre Antikörper, ANCA und Serumproteine regelrecht, negative Kryoglobulinbestimmung, keine Komplementdepletion. In der Serumelektrophorese konnten IgM-Lambda-Leichtketten (M-Gradient 1,4 g/l) nachgewiesen werden. Regelrechter Urinstatus.

Serologie. Antistreptolysin- und Antistreptodornase-Werte waren unauffällig, ein Westernblot für Borrelien diskret positiv. Die Serologien auf HIV, CMV, Brucellen, Francisella, Q-Fieber, Salmonellen, Toxoplasmose, EBV-IgM, TPHA, Adenovirus, Herpes simplex, Enteroviren, Parvovirus B19, Mycoplasma pneumoniae sowie HBV und HCV waren negativ. Es konnten positive HAV-Antikörper bei negativem IgM nachgewiesen werden. Kein bakterielles Wachstum in den Blutkulturen.

Knochenmarkpunktion. Unspezifische reaktive Veränderungen mit lymphoplasmozytoider Hyperplasie, vereinbar mit einer monoklonalen Gammopathie unbekannter Relevanz. In der Immunphänotypisierung kein Hinweis auf lymphoproliferativen Prozess im Knochenmark.

Hautbiopsie (Oberschenkel). Im Korium (Dermis) zeigten sich perivaskuläre und interstitielle, entzündliche Infiltrate aus Neutrophilen, Lymphozyten, Histiozyten und wenigen Eosinophilen. In der Umgebung der Gefäße fanden sich neben zahlreichen Neutrophilen auch wenige Kerntrümmer als Merkmal einer Leukozytoklasie (• Abb. 1). Die Gefäßwände wiesen eine Schwellung auf und waren von neutrophilen Granulozyten durchsetzt (- Abb. 2). Dieser Befund war vereinbar mit einer Urtikaria-Vaskulitis im Frühstadium.

\section{Diagnose}

- Schnitzler-Syndrom.

\section{Therapie und Verlauf}

Eine Behandlung mit Colchicin, Prednison, Ibuprofen und Fexofenadin wurde 2001 eingeleitet. Trotzdem berichtete der Patient über rezidivierende Schübe einmal wöchentlich mit Fieber, starkem Pruritus und beeinträchtigenden Gelenkschmerzen. Nach Wechsel von Colchicin auf Dapson traten die Anfälle alle $14 \mathrm{Ta}$ ge auf, wobei die Urtikaria weniger ausgeprägt war, die Arthralgien und das Fieber aber noch bestanden. Im Intervall war der Patient weitgehend beschwerdefrei. Trotz der Limitierung während der Anfälle lehnte der Patient eine Änderung der Therapie ab.

\section{Diskussion}

\section{Schnitzler-Syndrom}

Die französische Dermatologin Liliane Schnitzler beschrieb 1974 ein Syndrom mit Urtikaria, Fieber, Knochenschmerzen bei Hyperostosis, Arthritiden und monoklonaler IgM-Gammopathie [9], zusätzlich Lymphknotenvergrößerungen sowie eine Hepato- und/oder Splenomegalie [8]. Die Hautveränderungen imponieren histologisch als Urtikaria-Vaskulitis mit intra- und perivaskulären, granulozytären und/oder lymphozytären Infiltraten in der oberen Dermis. Die Knochenveränderungen sind häufig in Femur, Tiba und im Beckenskelett lokalisiert.

Die Erkrankung verläuft chronisch, bisher wurde keine Remission beschrieben. Die monoklonale IgM-Gammopathie und das Exanthem definieren dieses Syndrom. Bei der Diagnosestellung finden 
sich vorwiegend IgM-Spiegel $<10 \mathrm{~g} / \mathrm{l}$, welche im Verlauf stabil bleiben oder progressiv ansteigen. Bei letzterem ist ein Morbus Waldenström auszuschließen [4].

Die Pathogenese des Schnitzler-Syndroms ebenso wie die Bedeutung der monoklonalen IgM-Komponente sind unklar. Möglicherweise können Paraproteine Komplement aktivieren [6]. Saurat et al. konnten bei 6 von 9 Patienten mit Schnitzler-Syndrom polyklonale IgG-Anti-Interleukin-1a-Antikörper nachweisen [7].

Zur medikamentösen Behandlung des Schnitzler-Syndroms werden u. a. nichtsteroidale Antirheumatika, Antihistaminika, Steroide, Colchicin oder Dapson eingesetzt. Allerdings stellt sich die Behandlung als schwierig und enttäuschend dar, da Antihistaminika das Exanthem nicht kontrollieren können und die Ergebnisse der Behandlung mit Neutrophilenmigrationsinhibitoren unbeständig sind [4]. Einen neuen Ansatz in der Behandlung des Schnitzler-Syndroms bieten Interleukin1-Rezeptorantagonisten [2].

Die Prognose des Schnitzler-Syndroms ist in der Regel gut und nimmt in der Mehrzahl der Fälle einen benignen Verlauf, einige Fälle können - wie Schnitzlers Originalfall - ein lymphoproliferatives Syndrom entwickeln $[5,10]$. Daher sind im Langzeitverlauf regelmäßige Kontrollen angezeigt. In der Literatur sind bislang etwa 60 Fälle eines Schnitzler-Syndroms beschrieben worden.

\section{Differenzialdiagnosen}

Eine chronisch rezidivierende Urtikaria mit Allgemeinsymptomen wie Fieber und Arthralgien lässt zunächst den Verdacht auf eine Urtikaria-Vaskulitis aufkommen. Die meisten Fälle einer Urtikaria-Vaskulitis sind idiopathischer Genese, sie können aber auch mit Autoimmunerkrankungen (insbesondere systemischer Lupus erythematodes und Sjögren-Syndrom), Infektionen und Malignomen assoziiert sein. Syndrome, die mit einer Urtikaria-Vaskulitis einhergehen können, sind beispielsweise das Schnitzler-, Muckle-Wells- oder Cogan-Syndrom [1].

Die hereditären periodischen Fiebersyndrome sind charakterisiert durch wiederholte Fieberschübe und eine lokalisierte Entzündung, welche v. a. Serosa und
Synovia betreffen. Als Beispiele sind das familiäre Mittelmeerfieber, das HyperIgD-Syndrom und das 1982 erstmalig beschriebene TNF-Rezeptor assoziierte periodische Fieber zu nennen [3].

In der Differenzialdiagnose muss das Schnitzler-Syndrom ferner gegen den Morbus Still des Erwachsenen, den systemischen Lupus erythematodes, die Kryoglobulinämie, Lymphome und den Morbus Waldenström abgegrenzt werden. Insbesondere bei Lymphomen und/oder Paraproteinämie können auch erworbene C1-Esterase-Inhibitor-Mängel auftreten, welche typischerweise ein Angioödem verursachen. Weiter abzugrenzen ist das POEMS-Syndrom, welches charakterisiert ist durch eine monoklonale Gammopathie, Polyneuropathie, Hautveränderungen (v. a. Hyperpigmentation), Organomegalie und endokrine Störungen [6].

\section{Fazit für die Praxis}

\section{Auf Grund der sehr unterschiedlichen Symptome werden die Patienten mit Schnitzler-Syndrom häufig von Kollegen verschiedener Fachrichtungen gesehen. Richtungweisend in der Diagnose ist, ne- ben dem Nachweis einer monoklonalen Gammopathie, die Hautbiopsie.}

\section{Korrespondenzadresse}

Dr. L.U. Zimmerli

Medizinische Poliklinik, Universitätsspital

Petersgraben 4, 4031 Basel

Schweiz

LZimmerli@uhbs.ch

Interessenkonflikt. Der korrespondierende Auto gibt an, dass kein Interessenkonflikt besteht.

\section{Literatur}

1. Davis MD, Brewer JD (2004) Urticarial vasculitis and hypocomplementemic urticarial vasculitis syndrome. Immunol Allergy Clin North Am 24: 183-213

2. De Koning HD, Bodar EJ, Simon A et al. (2006) Beneficial response to anakinra and thalidomide in Schnitzler's syndrome. Ann Rheum Dis: 65: 542544

3. Drenth JP, Meer JW van der (2001) Hereditary periodic fever. N Engl J Med 345: 1748-1757

4. Lipsker D, Veran Y, Grunenberger F et al. (2001) The Schnitzler syndrome. Four new cases and review of the literature. Medicine 80: 37-44

5. Machet L, Vaillant L, Machet MC et al. (1996) Schnitzler's syndrome (urticaria and macroglobulinemia). Evolution to Waldenström's disease is not uncommon. Acta Derm Venereol 76: 413
6. Nashan D, Sunderkotter C, Bonsmann G et al. (1995) Chronic urticaria, arthralgia, raised erythrocyte sedimentation rate and IgG paraproteinaemia: a variant of Schnitzler's syndrome? Br J Dermatol 133: 132-134

7. Saurat JH, Schifferli J, Steiger G et al. (1991) Anti-interleukin-1 alpha autoantibodies in humans: characterization, isotype distribution, and receptor-binding inhibition - higher frequency in Schnitzler's syndrome (urticaria and macroglobulinemia). J Allergy Clin Immunol 88: 244-256

8. Schartz NE, Buder S, Sperl H et al. (2002) Report of a case of Schnitzler's syndrome treated successfully with interferon alpha 2b. Dermatology 205: 5456

9. Schnitzler L, Schubert B, Boasson J et al. (1974) Urticaire chronique, lésions osseuses, macroglobulinémie IgM: maladie de Waldenström? Bull Soc Dermatol Syphiligr 81: 363

10. Verret JL, Leclech C, Rousselet MC et al. (1993) Syndrome de Schnitzler et maladie de Waldenström. Evolution terminale du cas princeps. Ann Dermatol Venereol 120: 459-460 